ISSN 0103-9954

\title{
EFEITO DA ASSOCIAÇÃO DE BAGAÇO DE CANA, DO TIPO E DO TEOR DE ADESIVO NA PRODUÇÃO DE PAINÉIS AGLOMERADOS
}

\author{
ASSOCIATION EFFECT OF SUGAR CANE BAGASSE, TYPE AND LEVELS OF \\ ADHESIVE ON PARTICLEBOARD PRODUCTION
}

Rafael Farinassi Mendes ${ }^{1}$ Lourival Marin Mendes ${ }^{2}$ José Benedito Guimarães Júnior ${ }^{3}$ Rosimeire Cavalcante dos Santos ${ }^{4}$ Antonia Amanda da Silva César ${ }^{1}$

\section{RESUMO}

Este trabalho teve como objetivo avaliar o efeito da associação de bagaço de cana, tipo e teor de adesivo nas propriedades físico-mecânicas de painéis aglomerados com madeira de Pinus spp. O delineamento experimental se constituiu de um esquema fatorial $3 \times 2 \times 3$, sendo três porcentagens de substituição de madeira de pinus por bagaço de cana - 25, 50 e 75\%; dois tipos de adesivos - fenol-formaldeído (FF) e ureia-formaldeído (UF); e três teores de adesivo $-6,9$ e $12 \%$. O ciclo de prensagem utilizado foi de pressão de $40 \mathrm{kgf} / \mathrm{cm}^{2}$, temperatura de $160^{\circ} \mathrm{C}$ para UF e de $180^{\circ} \mathrm{C}$ para FF, e por um tempo de 8 minutos. Mediante os resultados pode-se concluir que: 1) Para a porcentagem de associação de bagaço de cana, nas propriedades em que foi observado efeito significativo, a tendência foi de diminuição da qualidade dos painéis à medida que se aumenta a quantidade de associação de bagaço de cana; 2) $\mathrm{O}$ adesivo UF se mostrou estatisticamente igual ou superior a FF em todas as propriedades avaliadas; 3) Os teores de adesivo apresentaram efeito significativo para todas as propriedades analisadas, sendo que, com exceção da propriedade ligação interna, em todas as outras propriedades o teor de $12 \%$ foi estatisticamente superior a $6 \%$, e ainda igual ou superior a $9 \%$.

Palavras-chave: aglomerados; bagaço de cana; pinus; tipo e teores de adesivo.

\begin{abstract}
This study aimed to evaluate the association effect of sugar cane bagasse, type and levels of adhesive in the physical-mechanical properties of particleboards with Pinus spp. wood. The experimental design consisted of a $3 \times 2 \times 3$ factorial scheme, with three percentages of substitution pine wood by sugar cane bagasse - 25 , 50 and $75 \%$; two types of adhesives - phenol-formaldehyde (PF) and urea-formaldehyde (UF); and three levels of adhesive $-6,9$ and $12 \%$. The cycle of pressing used was $40 \mathrm{kgf} / \mathrm{cm}^{2}$ pressure, temperature of $160^{\circ} \mathrm{C}$ for UF and $180^{\circ} \mathrm{C}$ for PF, and for a time of 8 minutes. From the results, it is concluded that: 1) For the percentage of sugar cane bagasse association, in the properties where significant effect was observed, the trend was decrease in panel quality as the amount of sugar cane bagasse increases; 2) The UF adhesive was statistically equal or greater than PF in all evaluated properties; 3) The levels of adhesive showed significant effects for all properties; Except for internal bond property, in all other properties the level of $12 \%$ was statistically superior to $6 \%$, and equal or greater than $9 \%$.
\end{abstract}

Keywords: particleboard; sugar cane bagasse; pine; type and adhesive levels.

1. Engenheiro Florestal, Doutorando em Ciência e Tecnologia da Madeira, Departamento de Engenharia Florestal, Universidade Federal de Lavras, Campus universitário, Caixa Postal 3037, CEP 37200-000, Lavras (MG). rafaelfarinassi@gmail.com / amanda ufla@hotmail.com

2. Engenheiro Florestal, Dr., Professor Adjunto do Departamento de Engenharia Florestal, Universidade Federal de Lavras, Caixa Postal 3037, CEP 37200-000, Lavras (MG). lourival@ufla.dcf.br

3. Engenheiro Florestal, Dr., Professor adjunto do Departamento de Engenharia, Universidade Federal do Piauí, BR 135, Km 03, CEP 64900-000, Bom Jesus (PI). jbguimaraesjr@hotmail.com

4. Engenheira Florestal, $\operatorname{Dr}^{\mathrm{a}}$ em Ciência e Tecnologia da Madeira, Departamento de Engenharia Florestal, Universidade Federal de Lavras, Campus universitário, Caixa Postal 3037, CEP 37200-000, Lavras (MG). meire_caico@yahoo.com.br

Recebido para publicação em 20/01/2010 e aceito em 20/12/2010 


\section{INTRODUÇÃO}

As vantagens tecnológicas dos painéis aglomerados são atribuídas principalmente à sua estrutura homogênea, possibilidade de utilização de matéria-prima sem restrições quanto à forma e às dimensões, e pelo fato de que, por meio do controle das variáveis de produção, pode-se adequar as propriedades físico-mecânicas do painel (IWAKIRI, 2005).

A principio os painéis podem ser produzidos a partir de qualquer material lignocelulósico que lhes confiram alta resistência mecânica e peso específico pré-estabelecido (ROWELL et al., 2000), os quais, após a aplicação de adesivo, se consolidam sob a ação de temperatura e pressão.

Atualmente, a indústria de painéis utiliza madeira de florestas plantadas, principalmente dos gêneros pinus e eucalipto, com certo destaque para o primeiro. No entanto, devido ao grande crescimento apresentado pelo setor, em função de fatores como: a modernização do parque fabril; o surgimento de novos produtos como o OSB, MDF e aglomerado/MDP; e pelo crescimento da construção civil e do setor de móveis, os quais são os principais consumidores; aumenta também a demanda por matéria-prima, o que leva à procura de outros tipos de materiais.

Neste contexto, o aproveitamento dos resíduos gerados pela agroindústria brasileira se mostra como alternativa para atender tal demanda de matéria-prima das indústrias de painéis aglomerados, apresentando-se com vários tipos de resíduos lignocelulósicos com potencialidades para aproveitamento, dentre os quais: sabugo de milho, casca de arroz, casca de café, casca de amendoim, caule de bananeira, casca de coco, caule de mandioca, casca de mamona, bagaço de cana, entre outros (MENDES, 2008a).

O bagaço de cana, que é um conjunto de fibras emaranhadas de celulose, tem sido produzido cada vez em maior quantidade, devido ao aumento da área plantada e da industrialização da cana-deaçúcar decorrentes de investimentos públicos e privados na produção alcooleira. Geralmente é queimado em reservatórios de vapor para produzir energia para uso industrial, mas atualmente devido às suas características físico-químicas, esta sendo utilizado em um vasto campo de pesquisas, dentre os quais: na produção de ração animal; na indústria química para a produção de plástico biodegradável; na produção de bicarbonato de sódio e cloreto de amônio e também para a manufatura de painéis aglomerados (ANSELMI, 2007).

Em questão da produção de painéis aglomerados, tal resíduo além de sofrer uma agregação de valor, pode ajudar a atender à demanda da indústria de painéis, o que ainda poderá causar uma redução nos custos de produção e consequentemente tornar o setor mais competitivo no cenário econômico.

Quanto às propriedades dos painéis, muitos são os fatores que contribuem para as características do produto final, dentre eles pode-se destacar: densidade do material lignocelulósico a ser utilizado, densidade dos painéis, umidade das partículas, razão de compactação, ciclo de prensagem, quantidade e tipo de adesivo (KELLY, 1977; MALONEY, 1993).

Dentre os principais tipos de adesivos utilizados pela indústria de painéis de madeira estão à ureia-formaldeído (UF) e fenol-formaldeído (FF). A UF apresenta resistência à umidade muito limitada, enquanto que o adesivo FF é indicado para produção de painéis para uso externo ou em ambiente com alta umidade relativa (IWAKIRI, 2005). Por ser o componente de maior custo, tornase importante a definição do tipo e quantidade de adesivo a ser utilizada, no sentido de se buscar uma otimização na relação custo/benefício.

Murakami et al. (1999) estudando o efeito dos teores de adesivo observaram um incremento nas propriedades mecânicas e na estabilidade dimensional dos painéis com o aumento do conteúdo de adesivo. Em contrapartida, Wu (1999) não encontrou uma relação bem definida entre teor de adesivo e valores de Módulo de elasticidade (MOE) e Módulo de ruptura (MOR), mencionando ainda que os efeitos do nível de adesivo sobre as propriedades dos painéis são relativos e não seguem uma tendência lógica.

Albuquerque (2002), estudando painéis aglomerados com densidades de 0,5;0,7 e $0,9 \mathrm{~g} /$ $\mathrm{cm}^{3}$, observou um aumento nos valores de ligação interna, flexão estática e inchamento em espessura, e uma diminuição dos valores de absorção de água, conforme se aumenta a densidade dos painéis. Em estudos realizados por Haselein (1989) e Zhow (1990), também foi observada uma relação linear positiva entre a densidade dos painéis e as propriedades de resistência à flexão estática (MOE e MOR) e à ligação interna.

A relação entre a densidade do painel e a densidade do material lignocelulósico, denominada de razão de compactação, exerce um efeito marcante 
nas propriedades dos painéis particulados, tendo em vista o processo de densificação do material para consolidação do painel até a espessura final. Kelly (1977) e Maloney (1993) recomendam uma razão de compactação na faixa de 1,3 a 1,6 , e relatam ainda que painéis de mesma densidade, quando produzidos com uma maior razão de compactação, apresentam normalmente maior resistência à flexão e à ligação interna, enquanto que as propriedades de absorção de água e inchamento em espessura passam a ter valores maiores. Em contrapartida, Vital et al. (1974) concluíram que painéis com maior razão de compactação $(1,6)$ apresentaram menor absorção de água que painéis com menor razão de compactação $(1,2)$, atribuindo tal fato à redução na porosidade dos painéis.

Mediante o exposto, o objetivo do trabalho foi avaliar o efeito da associação de bagaço de cana, tipo e teor de adesivo nas propriedades físicomecânicas de painéis aglomerados com madeira de Pinus spp.

\section{MATERIAL E MÉTODOS}

O bagaço de cana foi obtido de um alambique de produção artesanal de cachaça, localizado na cidade de Lavras, sul do estado de Minas Gerais. Já a madeira de Pinus spp. foi proveniente de plantios experimentais localizado no campus da Universidade Federal de Lavras UFLA, Lavras -MG.

O bagaço de cana foi utilizado da mesma forma em que foi coletado no alambique, ou seja, sem que houvesse a retirada da medula. Enquanto que as toras de madeira de Pinus spp. foram desdobradas em tábuas com espessura nominal de $25 \mathrm{~mm}$, mediante a utilização de uma serra de fita, e então, com o auxílio de uma serra circular, transformadas em blocos.

Os blocos de madeira e o bagaço de cana foram então triturados em um moinho martelo, com uma peneira de abertura de $6 \mathrm{~mm}$, para a geração das partículas do tipo sliver. Estas partículas foram secas a uma umidade de $3 \%$, com a utilização de uma estufa de circulação forçada de ar. Seguindo para uma encoladeira, onde foi aplicado o adesivo de acordo com o delineamento experimental demonstrado na Tabela 1, e também $1 \%$ de parafina (base peso seco das partículas) para todos os tratamentos. Para cada tratamento foram produzidos três painéis com densidade nominal de $0,70 \mathrm{~g} / \mathrm{cm}^{3}$.
TABELA 1: Delineamento experimental.

TABLE 1: Experimental design.

\begin{tabular}{|c|c|c|c|c|}
\hline \multirow[b]{2}{*}{ Tratamentos } & \multicolumn{2}{|c|}{ Associação de material } & \multicolumn{2}{|l|}{ Adesivo } \\
\hline & $\begin{array}{c}\text { Bagaço de } \\
\text { cana } \\
(\%)\end{array}$ & $\begin{array}{c}\text { Pinus } \\
\text { spp. } \\
(\%)\end{array}$ & Tipo & $\%$ \\
\hline $\mathrm{T} 1$ & 75 & 25 & \multirow{9}{*}{$\begin{array}{l}\text { Fenol- } \\
\text { formaldeído }\end{array}$} & 6 \\
\hline $\mathrm{T} 2$ & 50 & 50 & & 6 \\
\hline T3 & 25 & 75 & & 6 \\
\hline $\mathrm{T} 4$ & 75 & 25 & & 9 \\
\hline T5 & 50 & 50 & & 9 \\
\hline T6 & 25 & 75 & & 9 \\
\hline $\mathrm{T} 7$ & 75 & 25 & & 12 \\
\hline $\mathrm{T} 8$ & 50 & 50 & & 12 \\
\hline T9 & 25 & 75 & & 12 \\
\hline T10 & 75 & 25 & \multirow{9}{*}{$\begin{array}{l}\text { Ureia- } \\
\text { formaldeído }\end{array}$} & 6 \\
\hline T11 & 50 & 50 & & 6 \\
\hline T12 & 25 & 75 & & 6 \\
\hline T13 & 75 & 25 & & 9 \\
\hline $\mathrm{T} 14$ & 50 & 50 & & 9 \\
\hline T15 & 25 & 75 & & 9 \\
\hline T16 & 75 & 25 & & 12 \\
\hline $\mathrm{T} 17$ & 50 & 50 & & 12 \\
\hline T18 & 25 & 75 & & 12 \\
\hline
\end{tabular}

Após encoladas, as partículas foram depositadas em uma caixa formadora de colchão, com dimensões de $48 \mathrm{~cm} \times 48 \mathrm{~cm}$, para a realização da préprensagem, a qual ocorreu em uma prensa manual a $8 \mathrm{kgf} / \mathrm{cm}^{2}$. Posteriormente, o colchão foi levado para uma prensagem a quente, com temperatura de $160^{\circ} \mathrm{C}$ para o adesivo ureia-formaldeído (UF) e $180^{\circ} \mathrm{C}$ para fenol-formaldeído (FF), pressão de $40 \mathrm{kgf} / \mathrm{cm}^{2}$ e por um tempo de 8 minutos.

Inicialmente, retirou-se cerca de $2 \mathrm{~cm}$ de cada extremidade dos painéis com a utilização de uma serra circular esquadrejadeira, e em seguida foram retirados os corpos de prova, com suas dimensões de acordo com os ensaios aplicados. Para as propriedades físicas (absorção de água e inchamento em espessura) foi empregada a norma ASTM - D1037-93 (2002); e para as propriedades mecânicas (módulos de ruptura e elasticidade na flexão estática) foi empregada a norma DIN 52362 (1982), e para ligação interna a norma ASTM D1037-93 (2002). Posteriormente os painéis foram colocados em câmara de climatização e mantidos a temperatura de $20 \pm 2{ }^{\circ} \mathrm{C}$ e umidade relativa de $65 \pm$ $5 \%$, até atingirem a umidade de equilíbrio. 
A densidade básica da madeira de pinus foi determinada de acordo com a norma NBR 11941 (2003), enquanto que a densidade básica do bagaço de cana foi realizada de acordo os procedimentos listados por Mendes (2008a). Já a densidade do material lignocelulósico (DML) que foi utilizado no cálculo da razão de compactação ( $\mathrm{RC}=$ densidade do painel/DML), foi obtida com a soma da porcentagem utilizada de cada material multiplicada pela sua densidade.

Foi considerado um experimento em delineamento inteiramente casualizado com 3 repetições, em que os tratamentos estavam arranjados em um esquema fatorial $3 \times 3 \times 2$ (três porcentagens de cana $-25,50$ e $75 \%$; três teores de adesivo - 6, 9 e $12 \%$; e dois tipos de adesivo fenol e ureia-formaldeído). Como foi observada diferença estatística entre a densidade aparente média de cada tratamento, realizou-se uma análise de correlação entre a densidade dos painéis e cada uma das propriedades. Para as propriedades em que se observou correlação significativa procedeuse a uma análise de covariância com as médias ajustadas para a densidade dos painéis, utilizando rotinas do software Statistical Analysis System
(SAS, 1999). Para a diferenciação dos tratamentos foi utilizado o teste de médias Tukey, com 5\% de significância.

\section{RESULTADOS E DISCUSSÃO}

\section{Densidade básica do material lignocelulósico, densidade aparente e razão de compactação dos painéis}

Os valores médios obtidos para a densidade básica da madeira de Pinus spp. e do bagaço de cana foram de 0,502 e $0,098 \mathrm{~g} / \mathrm{cm}^{3}$, respectivamente. Já os valores médios da densidade aparente $(12 \%)$ e da razão de compactação dos diferentes tratamentos estão apresentados na Tabela 2.

A densidade aparente dos painéis variou na faixa de 0,610 a $0,670 \mathrm{~g} / \mathrm{cm}^{3}$. Estando, desta forma, todos os tratamentos classificados como painéis de média densidade, que se referem a painéis com densidade entre 0,59 e $0,80 \mathrm{~g} / \mathrm{cm}^{3}$ (IWAKIRI, 2005). Os baixos valores de densidade observados, tendo como base àquela nominal $\left(0,70 \mathrm{~g} / \mathrm{cm}^{3}\right)$, podem ser atribuídos à especificidade das condições laboratoriais em relação ao processo industrial, com perdas de materiais durante o

TABELA 2: Valores médios da densidade aparente e razão de compactação dos painéis.

TABLE 2: Average values of apparent density and compaction ratio of the panels.

\begin{tabular}{cccccc}
\hline \multicolumn{2}{c}{ Associação de material } & \multicolumn{2}{c}{ Adesivo } & $\begin{array}{c}\text { Densidade aparente } \\
\left(\mathrm{g} / \mathrm{cm}^{3}\right)\end{array}$ & RC=(DP/DML) \\
\hline Bagaço de cana (\%) & Pinus $(\%)$ & Tipo & $\%$ & 0,635 & 3,20 \\
50 & 25 & & 6 & 0,632 & 2,11 \\
25 & 50 & & 6 & 0,619 & 1,55 \\
75 & 75 & & 6 & 0,649 & 3,27 \\
50 & 25 & & 9 & 0,646 & 2,16 \\
25 & 50 & FF & 9 & 0,610 & 1,53 \\
75 & 75 & & 9 & 0,649 & 3,27 \\
50 & 25 & & 12 & 0,615 & 2,06 \\
25 & 50 & & 12 & 0,613 & 1,53 \\
75 & 75 & 12 & 0,639 & 3,22 \\
50 & 25 & & 6 & 0,670 & 2,24 \\
25 & 50 & & 6 & 0,635 & 1,59 \\
75 & 75 & & 6 & 0,635 & 3,20 \\
50 & 25 & & 9 & 0,645 & 2,16 \\
25 & 50 & UF & 9 & 0,649 & 1,62 \\
75 & 75 & & 9 & 0,649 & 3,27 \\
50 & 25 & & 12 & 0,637 & 2,13 \\
25 & 50 & & 12 & 0,656 & 1,64 \\
\hline
\end{tabular}

Em que: $\mathrm{RC}=$ Razão de compactação; $\mathrm{DP}=$ Densidade dos painéis; e $\mathrm{DML}=$ Densidade do material lignocelulósico 
manuseio das partículas nas etapas de aplicação de adesivo, formação do colchão e prensagem dos painéis.

Apenas os painéis produzidos com $25 \%$ de bagaço de cana apresentaram valores médios de razão de compactação estipulados como ideais por Kelly (1977) e Maloney (1993), que é na faixa de 1,3 a 1,6. Tais resultados se devem a baixa densidade do material bagaço de cana $\left(0,098 \mathrm{~g} / \mathrm{cm}^{3}\right) \mathrm{em}$ comparação com a densidade da madeira de pinus $\left(0,502 \mathrm{~g} / \mathrm{cm}^{3}\right)$, e como a razão de compactação é determinada pela relação entre a densidade do painel sobre a densidade do material lignocelulósico, tal valor aumenta conforme é adicionado maior porcentagem de bagaço de cana na associação com a madeira de pinus.

\section{Propriedades físicas \\ Absorção de água (AA)}

De acordo com a análise de variância para absorção de água após 2 e 24 horas de imersão (AA2h e AA24h), os resultados experimentais mostram que os resíduos seguem uma distribuição normal, segundo o teste de Shapiro-Wilk ( $\mathrm{p}$-valor $=$ 0,1641 e 0,2406 , respectivamente), e que a regressão linear não se mostrou significativa para $\mathrm{AA} 2 \mathrm{~h}$ $(\mathrm{p}$-valor $=0,0898)$ indicando que não existe uma relação linear entre essa propriedade e a densidade dos painéis. Enquanto que para AA24h, a regressão linear se mostrou significativa ( $\mathrm{p}$-valor $=0,0166$ ), com a estimativa do coeficiente de regressão linear indicando que para o aumento de $0,01 \mathrm{~g} / \mathrm{cm}^{3}$ na densidade dos painéis há uma redução de $0,90 \%$ na absorção de água.

Não foi observada interação entre as diferentes variáveis de produção (tipo de adesivo, teores de adesivo e porcentagem de associação de bagaço de cana).

$\mathrm{Na}$ análise das diferentes porcentagens de associação de bagaço de cana com a madeira de pinus (Tabela 3), houve efeito significativo dos tratamentos para AA2h e também para AA24h. Para $\mathrm{AA} 2 \mathrm{~h}$, os valores médios aumentam à medida que se aumenta a porcentagem de bagaço de cana, sendo todos os tratamentos diferentes estatisticamente. Enquanto que para AA24h, os painéis com 50 e $75 \%$ de bagaço de cana se mostraram estatisticamente iguais, mas diferentes estatisticamente dos painéis com $25 \%$ de bagaço de cana, os quais absorveram menor quantidade de água.

Essa tendência do aumento da absorção de água (AA2h e AA24h) conforme se aumentou
TABELA 3: Valores médios de absorção de água em função da porcentagem de bagaço de cana.

TABLE 3: Average water absorption values according to the percentage of sugar cane bagasse.

\begin{tabular}{ccc}
\hline Bagaço de cana & AA2h $(\%)$ & AA24h $(\%)$ \\
\hline $25 \%$ & $32,07(1,86) \mathrm{a}$ & $49,82(1,73) \mathrm{a}$ \\
$50 \%$ & $45,61(1,85) \mathrm{b}$ & $60,29(1,72) \mathrm{b}$ \\
$75 \%$ & $54,09(1,89) \mathrm{c}$ & $64,17(1,75) \mathrm{b}$ \\
\hline $\mathrm{CV}(\%)$ & 17,82 & 12,49 \\
\hline
\end{tabular}

Em que: Médias seguidas de mesma letra minúscula na coluna não diferem entre si pelo teste Tukey com um nível nominal de significância de 5\%. Dentro dos parênteses encontra-se o erro padrão.

a porcentagem de bagaço de cana se deve ao fato de que como o bagaço de cana apresenta menor densidade, o aumento de sua porcentagem causa a necessidade de um número maior de partículas para se obter a densidade pré-determinada do painel, ocasionando assim a disponibilidade de um número maior de sítios de sorção e por consequência o aumento da absorção de água.

Para as duas propriedades $(\mathrm{AA} 2 \mathrm{~h} \mathrm{e}$ AA24h), não houve diferença estatística para o tipo de adesivo, e em questão dos teores de adesivo, 9 e $12 \%$ se mostraram estatisticamente iguais, mas diferentes de $6 \%$, o qual obteve os maiores valores de absorção (Tabela 4).

A diminuição da absorção de água conforme se aumentou o teor de adesivo está relacionada a um maior recobrimento das partículas, fazendo com que ocasione uma melhor ligação das mesmas e também proporcione menor disponibilidade dos

TABELA 4: Valores médios de absorção de água em função do teor de adesivo.

TABLE 4: Average water absorption values according to the adhesive levels.

\begin{tabular}{ccc}
\hline Teor de adesivo & AA2h (\%) & AA24h $(\%)$ \\
\hline $6 \%$ & $55,56(1,84) \mathrm{b}$ & $68,61(1,71) \mathrm{b}$ \\
$9 \%$ & $37,15(1,85) \mathrm{a}$ & $53,56(1,71) \mathrm{a}$ \\
$12 \%$ & $39,06(1,85) \mathrm{a}$ & $52,12(1,71) \mathrm{a}$ \\
\hline $\mathrm{CV}(\%)$ & 17,82 & 12,49 \\
\hline
\end{tabular}

Em que: Médias seguidas de mesma letra minúscula na coluna não diferem entre si pelo teste Tukey com um nível nominal de significância de 5\%. Dentro dos parênteses encontra-se o erro padrão. 
sítios de sorção para a ligação com as moléculas de água, tendo por consequência a diminuição dos valores médios desta propriedade.

Iwakiri et al. (2001) avaliando o potencial de utilização de cinco espécies de pinus tropicais para produção de painéis aglomerados, e sem a utilização de parafina, encontraram valores médios de absorção de água após 2 e 24 horas de imersão variando na faixa de 68,82 a $83,60 \%$ e de 78,69 a $93,8 \%$, respectivamente.

Mendes et al. (2010) avaliando a associação das madeiras de eucalipto e pinus na produção de painéis aglomerados de bagaço de cana obtiveram, para os painéis de bagaço de cana, valores médios de AA2h e AA24h de 57,79 e 77,38, respectivamente.

Neste sentido, os painéis de bagaço de cana produzidos em associação com madeira de pinus apresentaram valores médios de AA2h e AA24h abaixo dos encontrados na literatura.

\section{Inchamento em espessura (IE)}

De acordo com a análise de variância para inchamento em espessura após 2 e 24 horas de imersão (IE2h e IE24h), os resultados experimentais mostram que os resíduos seguem uma distribuição normal, segundo o teste de Shapiro-Wilk ( $\mathrm{p}$-valor $=0,4705$ e 0,2113, respectivamente), e que a regressão linear não se mostrou significativa para as duas propriedades ( $\mathrm{p}$-valor $=0,9966$ e 0,0847 , respectivamente) indicando que não existe uma relação linear entre essas propriedades e a densidade dos painéis.

Não foi observada interação entre o tipo de adesivo, teores de adesivo e a porcentagem de associação de bagaço de cana.

De acordo com os resultados, observa-se que existe um efeito significativo das porcentagens de associação de bagaço de cana com a madeira de pinus para a propriedade IE2h, sendo 25 e $50 \%$ de bagaço de cana estatisticamente iguais, mas diferentes de $75 \%$, o qual apresentou os maiores valores de inchamento (Tabela 5). Para IE24h, esse efeito significativo não foi observado (Tabela 5).

Para o tipo de adesivo, não houve diferença estatística para IE2h e também para IE24h. Enquanto que para os teores de adesivo, todos diferenciaram estatisticamente, sendo $12 \%$ o que apresentou menor inchamento em espessura, tanto para 2 como para 24 horas (Tabela 6). Tal fato é explicado por uma maior quantidade de adesivo por partícula, o que causa uma melhor ligação entre as mesmas, acarretando em um menor inchamento em espessura.
TABELA 5: Valores médios de inchamento em espessura em função das porcentagens de bagaço de cana.

TABLE 5: Average thickness swelling values according to the percentage of sugar cane bagasse.

\begin{tabular}{ccc}
\hline $\begin{array}{c}\text { Porcentagem } \\
\text { bagaço de cana }\end{array}$ & IE2h (\%) & IE24h (\%) \\
\hline $25 \%$ & $7,82(0,42) \mathrm{b}$ & $11,70(0,42) \mathrm{a}$ \\
$50 \%$ & $9,08(0,41) \mathrm{b}$ & $12,90(0,42) \mathrm{a}$ \\
$75 \%$ & $10,55(0,42) \mathrm{a}$ & $13,42(0,43) \mathrm{a}$ \\
\hline $\mathrm{CV}(\%)$ & 19,15 & 10,76 \\
\hline
\end{tabular}

Em que: Médias seguidas de mesma letra minúscula na coluna não diferem entre si pelo teste Tukey com um nível nominal de significância de 5\%. Dentro dos parênteses encontra-se o erro padrão.

TABELA 6: Valores médios de inchamento em espessura em função dos teores de adesivo.

TABLE 6: Average thickness swelling values according to the adhesive levels.

\begin{tabular}{ccc}
\hline Teor de adesivo & IE2h (\%) & IE24h (\%) \\
\hline $6 \%$ & $14,92(0,41)$ a & $18,88(0,42) \mathrm{a}$ \\
$9 \%$ & $7,33(0,41) \mathrm{b}$ & $11,04(0,42) \mathrm{b}$ \\
$12 \%$ & $5,20(0,41) \mathrm{c}$ & $8,10(0,42) \mathrm{c}$ \\
\hline $\mathrm{CV}(\%)$ & 19,15 & 10,76 \\
\hline
\end{tabular}

Em que: Médias seguidas de mesma letra minúscula na coluna não diferem entre si pelo teste Tukey com um nível nominal de significância de 5\%. Dentro dos parênteses encontra-se o erro padrão.

Iwakiri et al. (2001) avaliando o potencial de utilização de cinco espécies de pinus tropicais para produção de chapas de madeira aglomerada, e sem a utilização de parafina, encontraram valores médios para inchamento em espessura após 2 e 24 horas de imersão em água variando na faixa de 25,20 a $34,80 \%$ e de 32,70 a $39,70 \%$, respectivamente. Desta forma, os painéis de bagaço de cana produzidos em associação com madeira de pinus apresentaram valores médios de inchamento em espessura a 2 e 24 horas abaixo dos encontrados na literatura.

A norma Commercial Standard - CS 23666 (1968) estipula valores máximos de inchamento em espessura a 24 horas de imersão de $35 \%$, tanto para UF como para FF, estando, assim, todos os tratamentos de acordo com a norma. 


\section{Propriedades mecânicas \\ Módulo de elasticidade (MOE) e módulo de ruptura (MOR)}

De acordo com a análise de variância para módulo de elasticidade (MOE) e módulo de ruptura (MOR), os resultados experimentais mostram que os resíduos seguem uma distribuição normal, segundo o teste de Shapiro-Wilk ( $\mathrm{p}$-valor $=0,7021$ e 0,5520 , respectivamente), e que a regressão linear foi significativa para as duas propriedades ( $p$-valores $<0,0001$ ) indicando que existe uma relação linear entre essas propriedades e a densidade dos painéis. Para MOE a estimativa do coeficiente de regressão linear indica que para o aumento de $0,01 \mathrm{~g} / \mathrm{cm}^{3}$ na densidade dos painéis há um aumento de 284,15 kgf/ $\mathrm{cm}^{2}$. Enquanto que para MOR, para um aumento de $0,01 \mathrm{~g} / \mathrm{cm}^{3}$ na densidade dos painéis há aumento de $2,96 \mathrm{kgf} / \mathrm{cm}^{2}$.

Não foi observada interação entre as variáveis de produção (tipo de adesivo, teores de adesivo e a porcentagem de associação de bagaço de cana).

Para o MOE não houve efeito significativo para as porcentagens de associação de bagaço de cana com a madeira de pinus (Tabela 7). Já para MOR, tal efeito se mostrou significativo, sendo a porcentagem de $25 \%$ estatisticamente superior às demais, as quais se mostraram estatisticamente iguais (Tabela 7).

Para o tipo de adesivo não houve diferença estatística para a propriedade MOE, enquanto que para MOR esta diferença foi observada, sendo a

TABELA 7: Valores médios de Módulo de elasticidade e Módulo de ruptura em função das porcentagens de bagaço de cana.

TABLE 7: Average values of Elastic Modulus and Rupture Modulus according to the percentage of sugar cane bagasse.

\begin{tabular}{ccc}
\hline $\begin{array}{c}\text { Porcentagem } \\
\text { Bagaço de } \\
\text { cana }\end{array}$ & $\begin{array}{c}\text { MOE } \\
\left(\mathrm{Kgf} / \mathrm{cm}^{2}\right)\end{array}$ & $\begin{array}{c}\text { MOR } \\
\left(\mathrm{Kgf} / \mathrm{cm}^{2}\right)\end{array}$ \\
\hline $25 \%$ & $8.257,15(223,80)$ a & $114,44(2,98) \mathrm{a}$ \\
$50 \%$ & $8.425,03(222,41)$ a & $103,83(2,97) \mathrm{b}$ \\
$75 \%$ & $8.397,58(227,27)$ a & $95,24(3,03) \mathrm{b}$ \\
\hline $\mathrm{CV}(\%)$ & 11,24 & 12,01 \\
\hline
\end{tabular}

Em que: Médias seguidas de mesma letra minúscula na coluna não diferem entre si pelo teste Tukey com um nível nominal de significância de 5\%. Dentro dos parênteses encontra-se o erro padrão.
UF estatisticamente superior a FF (Tabela 8). Em questão dos teores de adesivo, houve diferença estatística entre todos os teores analisados tanto para MOE como para MOR, sendo $12 \%$ estatisticamente superior nas duas propriedades (Tabela 9).

Mendes et al. (2008b) comparando painéis comerciais produzidos com bagaço de cana na China com os painéis comerciais produzidos no Brasil com a madeira de eucalipto e pinus obtiveram valores médios para o MOE de $16400,16 \mathrm{kgf} / \mathrm{cm}^{2}$ para os painéis de pinus e de $16561,20 \mathrm{kgf} / \mathrm{cm}^{2}$ para os de bagaço de cana. Enquanto que para MOR os valores médios foram de $208,88 \mathrm{kgf} / \mathrm{cm}^{2}$ para pinus e $218,03 \mathrm{kgf} / \mathrm{cm}^{2}$ para bagaço de cana.

A norma CS 236-66 estipula valores mínimos para MOE de $24500 \mathrm{kgf} / \mathrm{cm}^{2}$ para o adesivo UF e de $17500 \mathrm{kgf} / \mathrm{cm}^{2}$ para FF. Para a propriedade

TABELA 8: Valores médios de Módulo de elasticidade e Módulo de ruptura em função dos tipos de adesivos.

TABLE 8: Average Elastic Modulus and Rupture Modulus values according to the adhesive types.

\begin{tabular}{ccc}
\hline $\begin{array}{c}\text { Tipo de } \\
\text { adesivo }\end{array}$ & MOE $\left(\mathrm{kgf} / \mathrm{cm}^{2}\right)$ & MOR $\left(\mathrm{kgf} / \mathrm{cm}^{2}\right)$ \\
\hline FF & $8.507,75(188,57) \mathrm{a}$ & $90,58(2,52) \mathrm{b}$ \\
$\mathrm{UF}$ & $8.212,09(188,57) \mathrm{a}$ & $118,43(2,52) \mathrm{a}$ \\
\hline $\mathrm{CV}(\%)$ & 11,24 & 12,01 \\
\hline
\end{tabular}

Em que: Médias seguidas de mesma letra minúscula na coluna não diferem entre si pelo teste $t$ de Student com um nível nominal de significância de 5\%. Dentro dos parênteses encontra-se o erro padrão.

TABELA 9: Valores médios de Módulo de elasticidade e Módulo de ruptura em função dos teores de adesivos.

TABLE 9: Average values of Elastic Modulus and Rupture Modulus according to the adhesive levels.

\begin{tabular}{ccc}
\hline Teor de adesivo & MOE $\left(\mathrm{kgf} / \mathrm{cm}^{2}\right)$ & MOR $\left(\mathrm{kgf} / \mathrm{cm}^{2}\right)$ \\
\hline $6 \%$ & $7.411,94(221,61) \mathrm{c}$ & $88,79(2,96) \mathrm{c}$ \\
$9 \%$ & $8.386,81(221,69) \mathrm{b}$ & $105,29(2,96) \mathrm{b}$ \\
$12 \%$ & $9.281,02(221,81) \mathrm{a}$ & $119,44(2,96) \mathrm{a}$ \\
\hline $\mathrm{CV}(\%)$ & 11,24 & 12,01 \\
\hline
\end{tabular}

Em que: Médias seguidas de mesma letra minúscula na coluna não diferem entre si pelo teste Tukey com um nível nominal de significância de 5\%. Dentro dos parênteses encontra-se o erro padrão. 
MOR os valores mínimos estipulados pela norma são de $112 \mathrm{kgf} / \mathrm{cm}^{2}$ para UF e de $126 \mathrm{kgf} / \mathrm{cm}^{2}$ para FF.

Diante disso, os painéis produzidos com bagaço de cana em associação com a madeira de pinus ficaram abaixo dos valores encontrados na literatura. E em comparação com a norma, os valores médios obtidos para o MOE ficaram abaixo do mínimo estipulado, o mesmo aconteceu para MOR com o adesivo FF. Já para MOR nos painéis produzidos com UF, apenas os com $25 \%$ de bagaço de cana e $12 \%$ de teor de adesivo atenderam a mesma.

Os baixos valores apresentados para MOE e MOR podem estar relacionados à baixa densidade do material bagaço de cana, o que resulta em um maior número de partículas para se obter uma densidade pré-determinada do painel e consequentemente na diminuição da quantidade de adesivo por partícula, proporcionando assim a diminuição dessas propriedades. Como também, pode ser em função da não retirada da medula do bagaço de cana, em que segundo estudos realizados por Hamid et al. (1983) e Atchinson \& Lengel (1985) a retirada da medula aumenta de 40 a $60 \%$ nas propriedades mecânicas dos painéis aglomerados.

\section{Compressão paralela}

De acordo com a análise de variância para compressão paralela, os resultados experimentais mostram que os resíduos seguem uma distribuição normal segundo o teste de Shapiro-Wilk (p-valor $=0,8594)$, e que a regressão linear significativa ( $p$-valor $<0,0001)$ indica que existe uma relação linear entre essa propriedade e a densidade dos painéis. A estimativa do coeficiente de regressão linear indica que para o aumento de $0,01 \mathrm{~g} / \mathrm{cm}^{3}$ na densidade dos painéis há um aumento de 3,12 kgf/ $\mathrm{cm}^{2}$ na compressão paralela.
Não foi observada interação entre o tipo de adesivo, teores de adesivo e a porcentagem de associação de bagaço de cana.

De acordo com os resultados não existe efeito significativo para a porcentagem de bagaço de cana associada com a madeira de pinus. Para o tipo de adesivo houve diferença estatística, sendo a UF estatisticamente superior a FF (Tabela 10). E para os teores de adesivo, $12 \%$ foi estatisticamente igual a $9 \%$ e ainda superior a $6 \%$, o qual se mostrou igual a 9\% (Tabela 10).

Mendes et al. (2008b) avaliando tipo e teores de adesivo na produção de painéis aglomerados de bagaço de cana, encontraram valores médios para compressão paralela variando na faixa de 22,97 a $33,71 \mathrm{kgf} / \mathrm{cm}^{2}$. Neste sentido os painéis produzidos com bagaço de cana em associação com a madeira de pinus apresentaram valores médios acima dos encontrados na literatura.

\section{Ligação interna}

De acordo com a análise de variância para ligação interna, os resultados experimentais mostram que os resíduos seguem uma distribuição normal segundo o teste de Shapiro-Wilk ( $\mathrm{p}$-valor $=0,6122)$, e que a regressão linear significativa $(p$-valor $=0,0229)$ indica que existe uma relação linear entre essa propriedade e a densidade dos painéis. A estimativa do coeficiente de regressão linear indica que para o aumento de $0,01 \mathrm{~g} / \mathrm{cm}^{3}$ na densidade dos painéis há uma redução de 0,40 kgf/ $\mathrm{cm}^{2}$ na ligação interna.

Foi observada interação entre o tipo de adesivo e a porcentagem de associação de bagaço de cana com a madeira de pinus, assim como entre o tipo de adesivo e os seus teores.

Para a interação entre o tipo de adesivo e a porcentagem de associação de bagaço de cana com a madeira de pinus observa-se que, dentro de cada porcentagem de associação de bagaço de

TABELA 10: Valores médios de compressão paralela em função dos tipos e teores de adesivos. TABLE 10: Average parallel compression values according to the types and adhesive levels.

\begin{tabular}{cccc}
\hline Tipo de adesivo & Compressão Paralela $\left(\mathrm{kgf} / \mathrm{cm}^{2}\right)$ & Teor de adesivo & Compressão Paralela $\left(\mathrm{kgf} / \mathrm{cm}^{2}\right)$ \\
\hline FF & $52,37(1,79) \mathrm{b}$ & $6 \%$ & $51,34(2,10) \mathrm{b}$ \\
$\mathrm{UF}$ & $60,50(1,79) \mathrm{a}$ & $9 \%$ & $56,64(2,10) \mathrm{ab}$ \\
& & $12 \%$ & $61,33(2,10) \mathrm{a}$ \\
\hline
\end{tabular}

CV (\%) 15,77

Em que: Médias seguidas de mesma letra minúscula na coluna não diferem entre si pelo teste Tukey com um nível nominal de significância de 5\%. Dentro dos parênteses encontra-se o erro padrão. 
cana avaliada, o adesivo ureia-formaldeído foi estatisticamente superior ao fenol-formaldeído. Enquanto que na análise do efeito da porcentagem de bagaço de cana dentro de cada tipo de adesivo, foi observada diferença estatística apenas para o adesivo ureia-formaldeído, sendo 25 e $50 \%$ iguais estatisticamente e superiores a $75 \%$ de bagaço de cana (Tabela 11).

$\mathrm{Na}$ interação entre o tipo de adesivo e seus teores, observa-se na análise dentro de cada teor de adesivo que a ureia-formaldeído também foi estatisticamente superior a fenol-formaldeído. E que para os diferentes teores dentro de cada tipo de adesivo, houve diferença estatística apenas para o adesivo ureia-formaldeído, sendo 12 e $9 \%$ iguais estatisticamente e diferentes de 6\% (Tabela 12).

TABELA 11: Valores médios de ligação interna em função das porcentagens de bagaço de cana e tipo de adesivo.

TABLE 11: Average internal bond values according to the percentage of sugar cane bagasse and adhesive types.

\begin{tabular}{ccc}
\hline Porcentagem & \multicolumn{2}{c}{ Tipo de adesivo } \\
\cline { 2 - 3 } Bagaço de cana & $\mathrm{FF}\left(\mathrm{kgf} / \mathrm{cm}^{2}\right)$ & UF $\left(\mathrm{kgf} / \mathrm{cm}^{2}\right)$ \\
\hline $25 \%$ & $5,41(0,37) \mathrm{b} \mathrm{A}$ & $7,74(0,33)$ a A \\
$50 \%$ & $5,02(0,33)$ b A & $8,57(0,34)$ a A \\
$75 \%$ & $4,04(0,33)$ b A & $5,66(0,33)$ a B \\
\hline
\end{tabular}

CV (\%) 13,55

Em que: Médias seguidas de mesma letra minúscula, na linha, e maiúscula, na coluna, não diferem entre si pelo teste Tukey com um nível nominal de significância de $5 \%$. Dentro dos parênteses encontra-se o erro padrão.

TABELA 12: Valores médios de ligação interna em função do tipo e teor de adesivo.

TABLE 12: Average internal bond values according to the types and adhesive levels.

\begin{tabular}{ccc}
\hline \multirow{2}{*}{ Teor de adesivo } & \multicolumn{3}{c}{ Tipo de adesivo } \\
\cline { 2 - 3 } & FF $\left(\mathrm{kgf} / \mathrm{cm}^{2}\right)$ & UF $\left(\mathrm{kgf} / \mathrm{cm}^{2}\right)$ \\
\hline $6 \%$ & $4,46(0,33) \mathrm{b} \mathrm{A}$ & $5,62(0,33)$ a B \\
$9 \%$ & $5,22(0,33) \mathrm{b} \mathrm{A}$ & $7,72(0,33)$ a A \\
$12 \%$ & $4,80(0,33) \mathrm{b} \mathrm{A}$ & $8,63(0,33)$ a A \\
\hline
\end{tabular}

CV (\%) 13,55

Em que: Médias seguidas de mesma letra minúscula, na linha, e maiúscula, na coluna, não diferem entre si pelo teste Tukey com um nível nominal de significância de $5 \%$. Dentro dos parênteses encontra-se o erro padrão.
Mendes et al. (2008b) comparando painéis comerciais produzidos com bagaço de cana na China com os painéis comerciais produzidos no Brasil com a madeira de eucalipto e pinus, obtiveram valores médios para ligação interna para bagaço de cana de $2,37 \mathrm{kgf} / \mathrm{cm}^{2}$ e para pinus de $5,49 \mathrm{kgf} / \mathrm{cm}^{2}$.

Mendes et al. (2010) avaliando a associação das madeiras de eucalipto e pinus na produção de painéis aglomerados de bagaço de cana obtiveram, para os painéis de bagaço de cana, valor médio de $1,96 \mathrm{kgf} / \mathrm{cm}^{2}$ para a propriedade ligação interna. Enquanto que para os painéis produzidos com $50 \%$ de pinus e $50 \%$ de bagaço de cana o valor médio obtido foi de $6,27 \mathrm{kgf} / \mathrm{cm}^{2}$

A norma CS 236-66 estipula valores mínimos para ligação interna de $4,2 \mathrm{kgf} / \mathrm{cm}^{2}$ para painéis produzidos com UF e FF.

Diante disso, os painéis produzidos neste estudo com bagaço de cana em associação com a madeira de pinus apresentaram valores acima dos encontrados na literatura para os painéis produzidos com bagaço de cana, e de acordo com os painéis produzidos com pinus e também com pinus em associação com bagaço de cana. Em comparação com a norma, apenas os painéis produzidos com $75 \%$ de bagaço de cana e adesivo FF não atenderam a mesma.

\section{CONCLUSÃO}

A porcentagem de associação de bagaço de cana na produção dos painéis de madeira de pinus não apresentou efeito significativo apenas para as propriedades inchamento em espessura após 24 horas de imersão, módulo de elasticidade e compressão paralela. Para a propriedade de ligação interna houve interação entre porcentagem de associação de bagaço de cana e tipo de adesivo, havendo diferença significativa apenas para os painéis produzidos com o adesivo fenol-formaldeído. Para os demais casos, a tendência foi de diminuição da qualidade dos painéis à medida que se aumenta a quantidade de associação de bagaço de cana;

$\mathrm{O}$ adesivo ureia-formaldeído foi estatisticamente igual ao fenol-formaldeído em todas as propriedades físicas avaliadas e também para o módulo de elasticidade, enquanto que nas demais propriedades foi estatisticamente superior;

Os teores de adesivo apresentaram efeito significativo para todas as propriedades analisadas, sendo que, com exceção da propriedade ligação interna para o adesivo fenol-formaldeído, em 
todas as outras propriedades o teor de $12 \%$ foi estatisticamente superior a $6 \%$. Em comparação com $9 \%$, o teor de adesivo de $12 \%$ foi estatisticamente superior apenas nas propriedades inchamento em espessura após 2 e 24 horas de imersão, módulo de elasticidade e módulo de ruptura, sendo igual estatisticamente nas demais.

\section{REFERÊNCIAS BIBLIOGRÁFICAS}

ALBUQUERQUE, C. E. C. Intervenções de variáveis no ciclo de prensagem de aglomerados. 2002. 150 f. Tese (Doutorado em Ciências Florestais)Universidade Federal do Paraná, Curitiba, 2002.

AMERICAN SOCIETY FOR TESTING AND MATERIALS.ASTM D-1037.Standard methods of evaluating properties of wood-base fiber and particles materials. Philladelphia.Annual book of ASTM Standard.v. 04.09. 2002.

ANSELMI, R. Diversificação industrial inclui bioeletricidade, levedura, plástico biodegradável e gás carbônico. Jornal cana, tecnologia industrial. Dez. 2007.

ASSOCIAÇÃO BRASILEIRA DE NORMAS TÉCNICAS- ABNT. Normas técnicas NBR 11941

- Madeira - Determinação da densidade básica. Rio de Janeiro: 2003. 6 p.

ATCHISON, J. E.; LENGEL D. E. Rapid growth in the use of bagasse as a raw material for reconstituted panel board. In: WASHINGTON STATE UNIVERSITY INTERNATIONAL PARTICLEBOARD/COMPOSITE MATERIAL SYMPOSIUM, 19., 1985, Pullman. Proceedings ... Pullmann, 1985. p. 145-193.

COMMERCIAL STANDARD.CS 236-66: mat formed wood particleboard. [S.I.], 1968.

HAMID, S. H., MAADHAH, A. G.; USANI, A. M. Bagasse-based building materials.Polym.Plast. Techol, Londres, v. 21, n. 2, p. 173-208, 1983.

HASELEIN, C. R. Análise de parâmetros para fabricação de chapas de partículas aglomeradas de madeira de eucalipto(Eucalyptusgrandis $w$. Hill ex-maiden) e embaúba (Cecropia sp.). 1989. 75 p. Dissertação (Mestrado em Ciências Florestais)Universidade Federal de Viçosa, Viçosa, 1989.

IWAKIRI, S. et al. Produção de chapas de madeira aglomerada de cinco espécies de pinus tropicais. Floresta e ambiente, v. 8, n. 1, p. 137 - 142, jan./dez. 2001.

IWAKIRI, S. Painéis de madeira reconstituída. Curitiba: FUPEF, 2005, 274 p.

KELLY, M. W. Critical literature review of relationships between processing parameters and physical properties of particleboard.USDA, for. Ser. Gen. Tech. Rep. FPL. Madison, v.10, 66 p., 1977.

MALONEY, T. M. Modern particleboard and dry-process fiberboard manufacturing. 2nd ed. São Francisco: M. Freeman, 1993. 689 p.

MENDES, R. F. Utilização do bagaço de cana de alambique na produção de painéis aglomerados. 2008.104 p. Monografia (graduação em Engenharia Florestal)-Universidade Federal de Lavras, Lavras, 2008a.

MENDES, R. F. et all. Qualidade de painéis aglomerados produzidos na China utilizando bagaço de cana. In: ENCONTRO BRASILEIRO EM MADEIRAS E EM ESTRUTURAS DE MADEIRA, 11., 2008, Londrina. Anais... Londrina, 2008.

MENDES, R. F.; MENDES, L. M.; ALMEIDA, N. F. Associação de eucalipto e pinus na produção de painéis aglomerados de bagaço de cana. In: ENCONTRO BRASILEIRO EM MADEIRAS E EM ESTRUTURAS DE MADEIRA, 12., 2010, Lavras. Anais... Lavras, 2010.

MURAKAMI, K. et al. Manufacture and properties of three-layered particleboards with oriented face strands of veneers I. Japan Wood Science, Tokyo, v. 45, n. 5 , p. 395-402, 1999.

NORMEN FÜR HOLZFASERPLATEN SPANPLATTEN SPERRHOLZ. DIN 52362. Testing of wood chipboards bending test, determination of bending strength. p. 39-40. 1982.

ROWELL, R. M.; HAN, J. S.; ROWELL, J. S. Characterization and Factors Affecting Fiber Properties.In: FROLLINI, E.; LEÃO, A. L.; MATTOSO, L. H. C. (eds.) Natural Polymersand Agrofibers Based Composites. São Carlos: Embrapa Instrumentação Agropecuária 2000 . p. 115134.

SAS Institute. SAS Procedures guide for computers. 6th ed. cary, NC, v.3, 373p. 1999.

VITAL, B. R., LEHMANN; BOONE, R. S. How species and board densities affect properties of exotic hardwood particleboards. Forest Products journal, Madison, v. 24, n. 12, p. 37-45, 1974.

WU, Q. In-plane dimensional stability of oriented strand panel: effect of processing variables. Wood andFiberScience. Madison, v. 31, n. 1, p. $28-40$. 1999.

ZHOW, D. A. Estudy of oriented structural board made from hybrid poplar. Phisical and mechanical properties of OSB. HolzAlsRoh Und Werkstoff, Berlin, v. 48, n. 7-8, p. 293-296, 1990. 